\title{
Design and Manufacturing of Cover Plate Parts
}

\author{
Xuezhong Wei \\ Weifang University of Science \& Technology, Shandong, Shouguang, 262700, China.
}

Keywords: Parts; Machine; Tool fixture; Process design.

\begin{abstract}
First of all, this thesis describes the cover parts of mechanical design part, including cover parts material, a cover plate parts of structure, process analysis, blank and the process dimension tolerance, covering parts processing route formulation, processing route, covering plate parts of machine tools and process equipment selection. Then it describes the design of the machine tool fixture design of the flat parts, including the basic requirements and the design procedure of the fixture.
\end{abstract}

\section{Introduction}

$\mathrm{NC}$ programming which is also known as NC machine tool machining program, is the most important thing part of the use of CNC machine tools. Programming and automatic programming of numerical control program. Manual programming is done by the manual tool path calculation and the preparation of the processing program. When the part shape is not very complicated or the machining program is not too long, manual programming is convenient and economy. Automatic programming is a computer through the automated programming software to complete the tool trajectory automatic calculation, automatic generation of machining program and on the computer screen dynamically show the tool path. For the complicated machining parts, especially when 3D solid shape or cutting, tool moving track is complicated. Automatic programming is adopted..

\section{Mechanical process design of cover plate parts}

Material selection for cover plate. Signs of the rationality of the material should meet the performance requirements of parts under the maximum play the potential of the materials, so it is necessary to consider the application of strength of materials to improve the level. It is also needed to reduce material consumption and reduce the proces sing cost. Therefore, to achieve equitable selection, designers must conduct comprehensive an aly sis and comprehensive consideration.

General under any of the following circumstances, materials are needed: design new products, changes in the original design or change parts of the original material cost reduction; in order to adapt to the factory on the condition of equipment and change the spare parts processing: the lack of raw materials and need to replacement of new materials. At present, most of the parts and tools are not stand ardized or not yet are standardized for selection of parts.

The general principle of material selection: firstly, it is the premise that the parts function is satisfied, considering the technology, economy, and according to the resources of our country, we choose the domestic material.

Structural process analysis of cover plate parts. The structural process analysis of the parts mainly includes three aspects, such as the dimension of the parts, the standard of the parts, the elements of the components and the structure of the parts..

The total length of spare parts is $300 \mathrm{~mm}$ and the width is $71.5 \mathrm{~mm}$. Tolerances is positive and negative is $0.05 \mathrm{~mm}$. The top view chart is shown in Fig. 1 . 


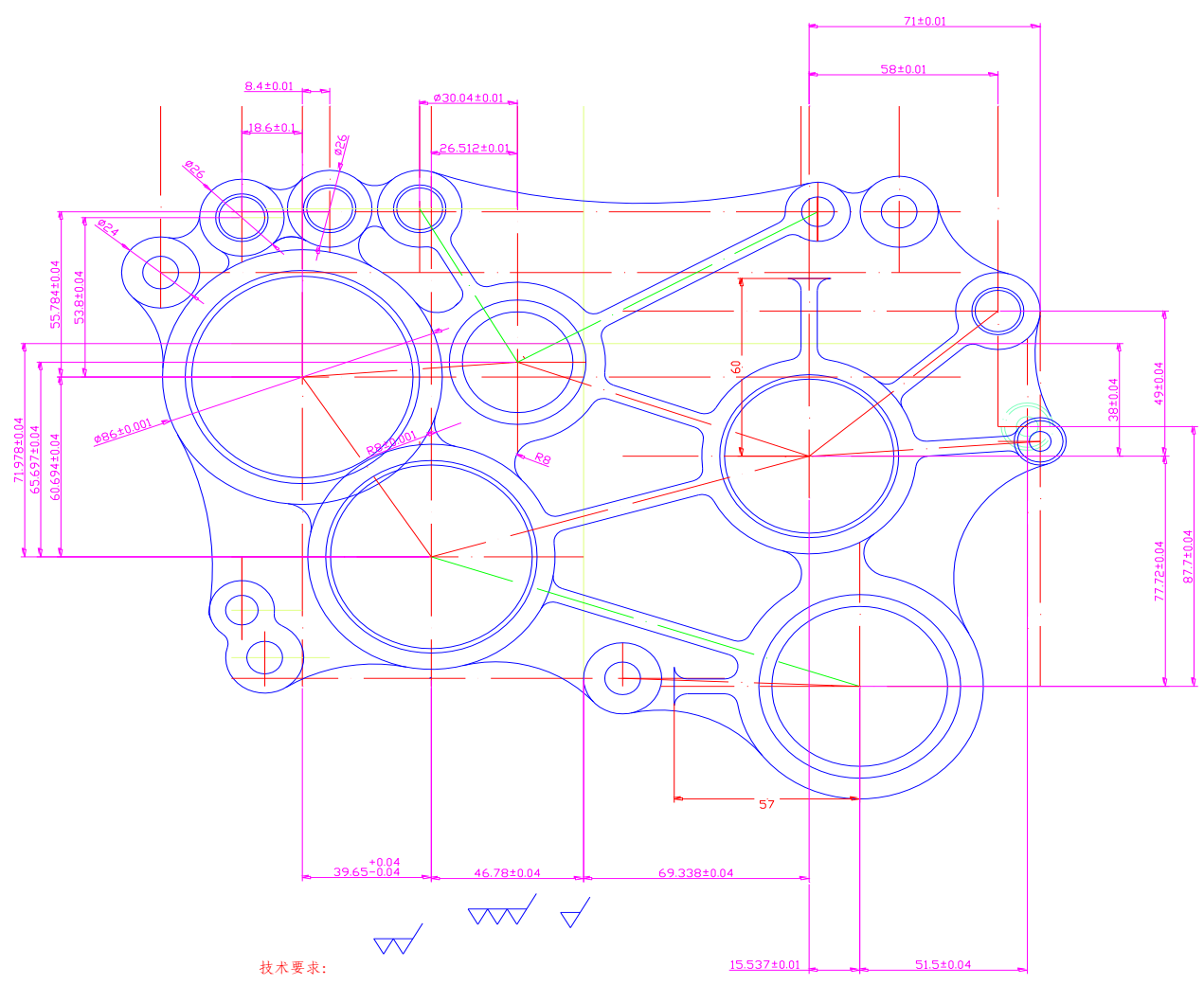

Fig. 1 Part size chart

Determination of tolerances for rough and working procedure dimensions. Size tolerances of Changzhou Jinguan agriculture (1729-214-043-OC) basic: boring tool is $0 \sim 0.03 \mathrm{~mm}$, drill is $0 \sim 0.3 \mathrm{~mm}$, size is $0 \sim 0.022 \mathrm{~mm}$, size and thickness is $-0.5 \sim+0.5 \mathrm{~mm}$.

The formulation of the processing route for the cover plate parts. Determination of process ing method

For milling on the workpiece, drilling, reaming, boring processing.

Processing sequence arrangement.

1) Rough machining: the main removal of the majority of the margin, so that the rough and size is close to the finished product, in the order of price creation conditions. In this part of the plane processing, 62mm, 72mm, Phi hole.

2) Finishing: guarantee the main surface to reach the drawing requirements. Finish machining datum, Phi 62mm, Phi 72mm rebore.

Centralization and decentralization of processes.

In the process of the processing of parts, it also needs to solve the problem of the centralized and the process of the process. Under different production conditions, the process of the preparation of the process is different.

How many of the processes of the same parts in the process are referred to the centralized and decentralized processes.

The process concentration is a lot of processing in each process, as far as possible in the installation of various surfaces, or as far as the same equipment on the continuous completion of more processing requirements. In this way, parts of the process is less, and the process is short.

The process is derived, the surface of the processing is very fine, each process of less content, the performance of the process, process route is long.

The process of concentration and dispersion has a characteristic, according to what principle to ascertain the number of processes, which to make comprehensive consideration according to the production program, machine tool equipment and parts of structure and technical requirements. But 
from the point of view of the development of technology, with the development and application of $\mathrm{CNC}$ machine tools, machining center, it will be more tend to focus on the process in the future.

Determination of processing order.

Arrangement of machining operation.

To meet all technical requirements of drawings and production with high efficiency and low cost, it does not only to correct selection of locating datum and each surface processing method, and reasonable arrangement procedure. This not only refers to the border between the mechanical machining, but also to reasonably arrange the order of the process between machining and heat treatment, surface treatment and as sistant working procedure (such as cleaning, inspection, etc.).

1) The first base. As other surface finishing benchmark general arrangement in the process of the beginning of processing.

2) After the Lord. Parts of the core surface (usually refers to the high machining accuracy and surface quality requirements of surface), the surface should first processing, thus early detection of defects that may appear in the blank.

3) First rough then finishes. A part of the cutting process, always rough first, then semi finishing, and finally finishing and finishing the finishing.

4) First hole. Box, bracket and other parts with contour dimen sions far than other surface size of the plane, is used as locating datum is stable and reliable. So most of the first processing of the plane as a fine benchmark, used for hole processing and other surfaces.

Determination of processing route. The process route means the movement track and direction of the relative workpiece in the process of the numerical control machine tools. That the pool from the starting point of the knife movement, until the point and the end of the process to return the path, including the introduction of cutting tools and cutting tools, return and other non - cutting air travel. Determine the machining path, mainly to determine the rough machining and air travel from the knife route, and the cutting process of the finishing cutting process is along the workpiece contour.

In determining the direction of machining, consider the tool "knife" phenomenon, especially in the numerical control milling machine and machining center of the cutting should beso. The milling route is usually used, and the milling route is employed in the milling process.

That is, milling the contour with the clockwise to the knife, milling the contour with the counterclockwise knife. The high speed machining center of the spindle over $10000 \mathrm{r} / \mathrm{min}$, to avoid the knife phenomenon caused by a knife.

Selection of machine tool and process equipment for flat parts. Selection of machine tools

Owing to the use of the tool when machining the part more, easy to take the wrong knife when milling machine, more process waste of resources, so the selection of machining center processing the workpiece.

Equipment choice.

Aperture measuring in diameter gauge and plug gauge; measuring flatness in marble platform with a feeler gauge; center distance in three dimen sion measuring other vernier caliper and micrometer.

\section{Machine tool fixture design for cover plate parts}

Basic require ments and design procedures for special fixture. Basic requirements for special fixture

1) Guarantee the machining accuracy of the workpiece

2) Improve productivity

3) Good craft

4) Good use

5) Good economy

Special jig design procedure.

1) Determination of positioning mode and selection of positioning element 
The positioning of the workpiece in the fixture is the most important process in fixture design, correct positioning of the only guarantee the workpiece to ensure the machining accuracy meets the requirements. The positioning fixture is to ensure process a batch of parts in the first part cutting until all processes are finished, the process workpiece reference relative to machine and tool always keep machining position correctly.

The workpiece in the fixture positioning to meet the six point location principle also guarantee the positioning accuracy of the workpiece, in the former process has finished two positioning holes and the bottom plane, so that a dual positioning can be used which is able to limit the work of six freedom degree requirements.

2) Determine the workpiece to inten sify the program, design and clamp device

Machining accuracy analy sis after the completion of the overall structure of the fixture elements, if the process precision of the workpiece has higher requirements, it should be established corresponding dimen sions, tolerances and position requirements fixture, and then the process accuracy analy sis.

a.Determination of tolerance

Because of the influence of various conditions, such as machine tool transmission error, fixture error, cutting tool error, human error, etc.. Cause the workpiece machining error. In order to guarantee the machining accuracy, it is a must to control the above error. In the development of fixture, the fixture error, error, error and adjustment error should be ensured, and the error formula is met, and the tolerance of the workpiece is generally $1 / 3$.

b.Analysis of working procedure precision

To examine the factors that cause the machining errors of the workpiece process, there are some errors: location error, installation error, adjustment error, machining error. In order to guarantee the machining accuracy of the workpiece must take measures to control and reduce the error caused by various factors, so that the machining error is not exceed ed the process dimension tolerance:

$$
\sum=\triangle \mathrm{D}+\triangle \mathrm{A}+\triangle \mathrm{T}+\triangle \mathrm{G} \leqslant \mathrm{g}
$$

The calculation formula (1) of error calculation formula, widely used in the analysis of the probability of proofreading.

For the analysis of the parts involved in the corresponding process dimension, the fixture design parameters and the manufacturing accuracy use better data to ensure machining precision, which will not be one by one calculation and accurate analysis of the process.

\section{Conclusions}

The development of numerical control technology. When to keep pace with the times, it is necessary to combine theory with practice. $\mathrm{CNC}$ automatic machining mode avoids the errors caused by the producer of a human operator, as well as the size of the same batch processing parts good consistency, high rate of qualified products, superior processing efficiency, greatly reduce the labor intensity of workers. CNC machine tool cannot only plane processing, but also can process the complicated curved surface parts; CNC machine tools are also conducive to the modemization of production management, with computer numerical control (CNC) machining, it is able to accurately estimate the working hours, with a precise formulation of the production schedule table.

\section{References}

[1] Junshan Xu. Application of numerical control technology in programming [J]2011.

[2] Yu Zhang, Ping Liu. Geometric tolerances and measurement technology. Shenyang: Northeastem University press 1999.

[3] Kun Wang. Mechanical design, mechanical design, basic curriculum design, Beijing: Higher Education Press, 1996. 
[4] Qiliang Guo. Course design of mech anical components, Guizhou people press, 1982.1.

[5] Kai Wang. Mechanical design standard application manual / second volume, Beijing: Machinery Industry Press, 1997.8.

[6] Lianggui Pu. Mechanical design (Seventh Edition), Beijing: Higher Education Press, 2001.

[7] Qing Zhang. The application of numerical control technology in mechanical manufacturing [J] Hun an agricultural machinery, 2011.

[8] Gao xiang Cong.[J]. CNC technology. High Boxiang cluster application and development prospect in the machining of the value engineering, 2011 (3). 